\title{
Description de la larve et de la nymphe de Leptoconops (Holoconops) kerteszi Kieffer, 1908 (Diptera, Ceratopogonidae)
}

\author{
par J. CLASTRIER \\ Laboratoire d'Ecologie médicale et de Pathologie parasitaire ( $\mathrm{P}^{\mathrm{r}}$ J.-A. Rıoux), \\ Faculté de Médecine, F. 34000 Montpellier \\ Institut national de la Santé et de la Recherche médicale
}

\begin{abstract}
Résumé
Description de la larve au $4^{e}$ stade et de la nymphe de Leptoconops $(H$.) kerteszi $\mathrm{K}$. Le squelette céphalique interne ainsi que diverses formations et sensilles de la face ventrale de la larve sont examinées. La chétotaxie du céphalothorax de la nymphe ressemble à celle des Culicoides. L'opercule et la trompette respiratoire sont de très petite taille; cette dernière est lisse et nue, et montre une double rangée de papilles terminales.
\end{abstract}

\section{Summary}

The fourth instar larva and the pupa of the biting midge Leptoconops $(H$.) kerteszi $K$. are described. The internal skeleton of the larval head as well as the structures and sensillae of its ventral face are examined. The chaetotaxy of the pupal cephalothorax resembles that of Culicoides. The operculum and the respiratory trumpets are very small; the latter is bare and smooth and shows a double row of distal papillae. 
La nuisance de Leptoconops (Holoconops) kerteszi Kieffer, 1908, dans le Midi de la France est moins grave que celle de son congénère $L$. (L.) irritans Noé, 1905, en raison de son apparition plus précoce (avril-mai) et de son extension limitée, mais elle n'en est pas moins cuisante. Le biotope d'élection de cette espèce a été précisé par J.-A. Rioux et S. Descous (1965), c'est la souillère. « Ce terme, couramment utilisé par les paysans et les manadiers de Petite-Camargue (inclus Bas-Gard), s'applique à des cuvettes sableuses de quelques hectares, noyées la majeure partie de l'année. » Le Laboratoire d'Ecologie médicale de la Faculté de Médecine de Montpellier a installé, il y a quelques années, une station d'observation sur l'une d'elles, dans le domaine privé de La Figueirasse. Nous y avons isolé à plusieurs reprises des larves et des nymphes de $L$. kerteszi et nous nous proposons de compléter la description de ces formes immatures précédemment donnée par L. M. Smith et H. Lowe (1948) d'après des exemplaires californiens. Ces larves vivent dans la couche superficielle de sable (de la surface à $5 \mathrm{~cm}$ de profondeur environ) au-dessus du niveau de l'eau de la souillère.

Larve ( $4^{\circ}$ stade).

La larve fraîchement extraite est d'une teinte saumonée, avec des variations allant de l'ambre à l'orangé ; conservée au laboratoire, elle pâlit légèrement, vraisemblablement sous l'effet d'une alimentation inadaptée et insuffisante. (A l'état normal, l'intestin contient un très grand nombre d'algues unicellulaires, principalement Navicules et Diatomées). Placée dans l'eau, sans particules solides pour y prendre appui, elle s'agite lentement, maladroitement, paraissant éprouver de grandes difficultés pour se déplacer. Si l'on ajoute à ce milieu liquide quelques brindilles végétales, elle s'y fixe plus ou moins fermement et les broute ; si les larves sont nombreuses, il arrive qu'elles se broutent mutuellement. Lors de l'extraction et les jours suivants, les larves se lovent lorsqu'elles sont dérangées, ressemblant alors à une columelle de Mollusque brisée, mais elles s'accoutument très rapidement aux manipulations dont elles peuvent être l'objet et ne paraissent plus s'en inquiéter. De temps à autre, sans raison apparente, elles se placent sur le dos, rectilignes, immobiles, ne réagissant pas, ou faiblement, au contact ou à la pression, paraissant mortes ou mourantes ; après un temps plus ou moins long, elles récupèrent une activité normale.

Cette larve est transparente, son anatomie interne visible avec la plus grande netteté. Après immersion dans l'alcool, elle s'opacifie et devient uniformément blanchâtre. Sa longueur est de $4 \mathrm{~mm}$ environ et son plus grand diamètre oscille autour de $250 \mu$, mais, placée sur un tamis, elle passe aisément à travers des mailles d'ouverture nettement inférieure en raison de sa très grande plasticité. Elle compte 23 segments (1 céphalique, 3 thoraciques, 19 abdominaux) parfaitement individualisés, qui sont beaucoup plus larges dans la partie moyenne du corps qu'à chacune de ses extrémités (fig. 3). A l'exception des deux premiers et du dernier, tous ces segments sont extérieurement semblables, de la même forme, de la même teinte, présentant de très fines stries transversales et sans autre accident tégumentaire qu'un nombre peu élevé d'éléments sensoriels. 
Conservant l'ordre et la terminologie précédemment suivis pour L irritans (J. Clastrier, 1971), nous verrons successivement : 1) le squelette céphalique ; 2) les formations sensorielles; 3) le segment anal.

\section{I. - SQuelette céphalique.}

Comme ses congénères, la larve de $L$. kerteszi est dépourvue de capsule céphalique hautement sclérifiée; elle possède en compensation un squelette céphalique interne d'aspect gracile, que l'on peut arbitrairement diviser, pour son étude, en 6 sections.

1) Squelette ventro-latéral (fig. 1, B, C) ; constitué par deux tiges longitudinales s'étendant depuis la partie postérieure du prothorax jusqu'au tiers antérieur du segment céphalique $(t v l)$; tiges rectilignes et légèrement convergentes de l'arrière vers l'avant dans leurs deux tiers postérieurs ; coudées vers la face dorsale et décrivant une courbe légère à concavité externe dans leur tiers antérieur. Leur base, médiocrement développée, est soudée avec l'extrémité postérieure du bras dorso-ventral correspondant (voir § 2) ; leur extrémité antérieure est appliquée sur le premier sclérite de l'arc génien (voir \$ 4).

2) Le squelette dorsal (fig. 1, A, B) comprend une tige impaire et médiane ( $\mathrm{tdm}$ ) mince, à bords irréguliers, d'aspect fragile. Son extrémité postérieure, ou base, se situe à la limite du segment céphalique ; elle est élargie et forme 2 ailerons. Chacun des ailerons est soudé avec un bras transversal, ou dorso-latéral ( $b d l)$, longuement coudé à angle droit à son extrémité externe, qui porte en outre un petit appendice ventral de forme assez variable. Ailerons et bras sont peu sclérifiés, à bords extrêmement irréguliers ; ils sont reliés par un dernier élément, le bras dorso-ventral $(b d v)$, à la tige ventrolatérale correspondante. L'extrémité antérieure de la tige dorso-médiane reste très éloignée des deux piliers frontaux.

3) Pilier frontal (fig. 1, A', B', C'). Cette partie du squelette est beaucoup plus simple que chez $L$. irritans. Le «noyau central \& ayant disparu, il subsiste seulement les trois apodèmes avec leurs mêmes caractéristiques, directement soudés les uns aux autres. Le premier, dit fronto-dorsal (afd), se dirige dorsalement en épousant la courbure du segment céphalique ; son extrémité reste fort éloignée, dans les sens longitudinal et transversal, de celle de la tige dorso-médiane. Le deuxième, fronto-ventral ( $a f v)$, presque rectiligne, vient s'appliquer sur l'extrémité antérieure du premier sclérite de l'arc génien (voir $\S 4$ ). Le troisième, fronto-médian $(\mathrm{afm})$, décrit une courbe vers la partie médiane de la tête, qui place son extrémité en position interne, antérieure et dorsale par rapport à celle du précédent. C'est dans la fourche parfaitement immobile formée par ces deux derniers apodèmes que s'articule la mandibule. L'apodème médian présente le même aspect que les deux autres ; c'est à seule fin de rendre son identification plus aisée et de permettre une meilleure comparaison avec $L$. irritans, qu'il a été ombré sur la figure.

4) L'arc génien (fig. 1, B, B', C, C') se présente de la même façon que chez $L$. irritans, en forme de lyre dont chacune des moitiés est composée de trois sclérites. Le sclérite le plus externe (agl) entre en contact, par sa courbure postérieure, avec l'extré- 
mité antérieure de la tige ventro-latérale correspondante. Il ne s'agit nullement d'une articulation véritable comportant épaulement et condyle, ni d'une soudure, mais d'une juxtaposition, assez lâche d'ailleurs. C'est ainsi qu'au cours de l'examen de larves vivantes anesthésiées, dans l'eau, il nous est arrivé plusieurs fois, au moment où la larve recommençait à bouger, d'observer l'ouverture répétée, brève mais complète, de cette jointure. Par l'extrémité antérieure du premier sclérite, l'arc génien immobilise la fourche articulaire et participe à cette articulation.

Le sclérite intermédiaire (ag2) est une lame courbe, à convexité externe, de direction générale dorso-ventrale, soudée dans sa partie moyenne au sclérite précédent (dont l'extrémité postérieure reste cependant libre) (fig. 1, E). Sa moitié dorsale (ag2d) se dirige vers l'hypopharynx ; sa moitié ventrale $(a g 2 v)$ représente la première courbure de l'S très serré décrit chez $L$. irritans.

Le troisième sclérite $(a g 3)$ forme le jambage et la deuxième courbure de l'S. Il ne touche pas son homologue du côté opposé, et dans l'espace resté libre vient se placer la partie la plus antérieure, effilée, du labium (lbi).

Signalons ici la présence, dans la partie latérale et postérieure de l'arc génien, d'une petite formation sclérifiée qui n'existe pas chez $L$. irritans. Elle se présente comme une esquille $(e s q)$ indépendante du reste du squelette, assez superficielle pour que suivant l'angle d'observation elle apparaisse au-dessous ou au-dessus de l'arc génien, mais néanmoins sous-tégumentaire.

5) Pièces buccales (fig. 1, B, B', C, C', F). Les mandibules (md) sont de taille moyenne, très largement séparées à la base, rapprochées dans leur partie moyenne et se faisant face par leur convexité. Elles portent trois dents, dont la plus externe, qui est aussi la plus petite, est recourbée en crochet. Leur bord interne subit un développement considérable et le condyle basal s'articule directement sur la fourche du pilier frontal. Le «sclérite innominé », décrit chez L. irritans n'a pas d'équivalent ici.

Elles fonctionnent verticalement et alternativement et possèdent une grande amplitude de mouvement. A l'ouverture, elles forment un angle de $135^{\circ}$ environ avec l'axe du corps, soit $45^{\circ}$ de plus qu'il n'est représenté en B et B'. En sens inverse, leur course est limitée par le diaphragme buccal (voir plus loin). On observe quelquefois, sur des exemplaires montés, des mandibules ayant plus ou moins largement franchi ce diaphragme et l'arc génien, comme sur la figure 1 en $\mathrm{A}^{\prime}$. Il s'agit vraisemblablement d'un artéfact provoqué par les variations de pression osmotique dont la larve est le siège lorsqu'elle est placée dans le milieu de montage, qui peuvent occasionner d'autres défor-

FIg. 1. - Larve de Leptoconops kerteszi K. A, vue dorsale ; B, vue latérale ; C, vue ventrale du segment céphalique, du prothorax et de la moitié antérieure du mésothorax. A': squelette de la bouche et du pharynx en vue fronto-dorsale (les mandibules ont largement dépassé l'arc génien). B' : vue latérale; C': vue fronto-ventrale du squelette de la bouche. D: vue frontale du pharynx. E: vue oblique interne de la moitié sroite de l'arc génien. F: mandibule gauche vue par sa face interne. afd, afm, afv: apodèmes fronto-dorsal, -médian, -ventral, ag1, ag2, ag3: $1^{\mathrm{er}}, 2^{*}, 3^{\mathrm{e}}$ sclérites de l'arc génien; $a g 2 d, a g 2 v$ : moitiés dorsale et ventrale du $2^{e}$ sclérite. an: antenne. bdl, bdv: bras dorsolatéral, -ventral. epi : épipharynx. esq: formation en esquille. hypo: hypopharynx. lbi: labium. md: mandibule. pmd: prémandibule. post: sclérites postérieurs de l'épipharynx. $t d m$ : tige dorso-médiane. $t v l$ : tige ventro-latérale 

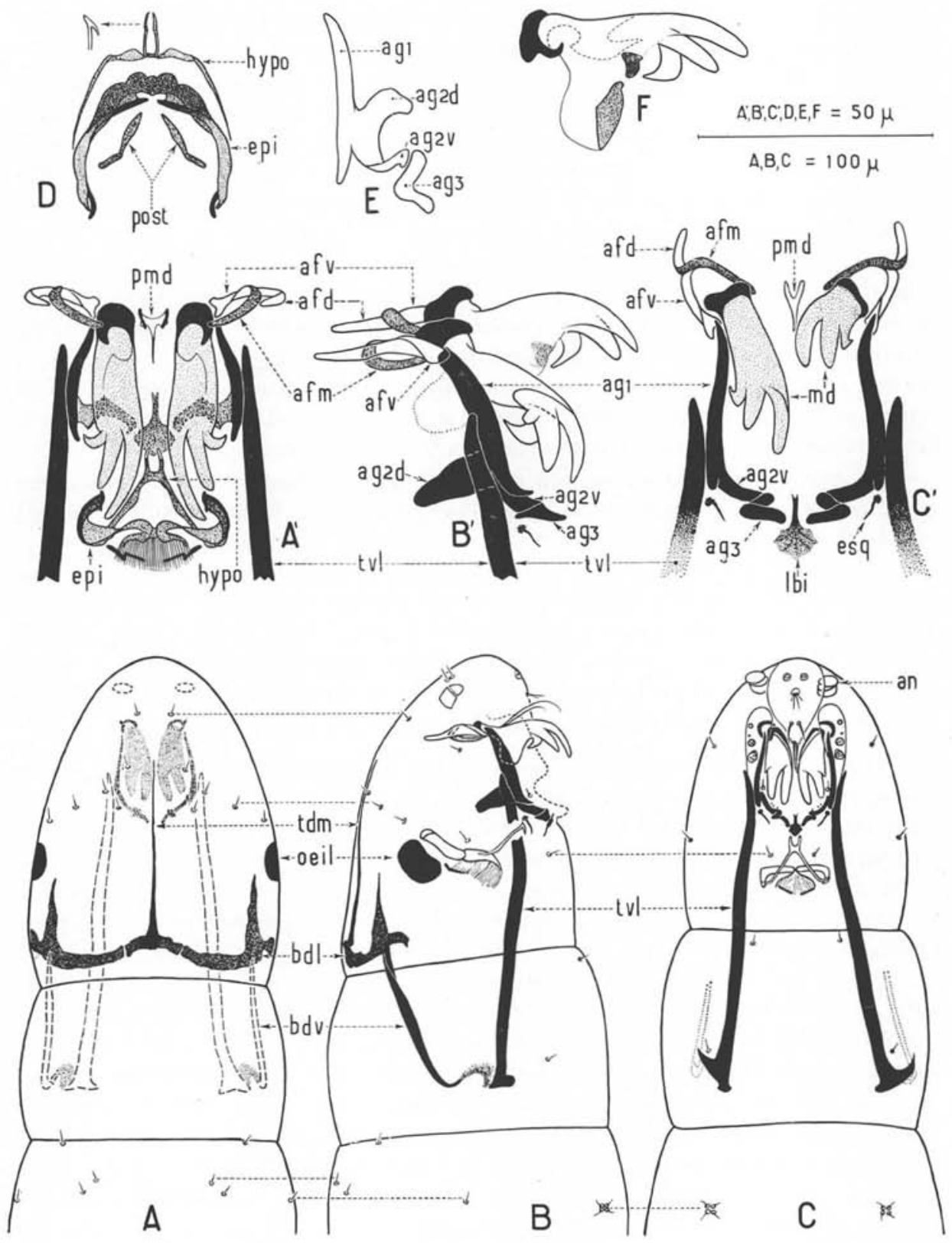
mations, parfois considérables, du squelette céphalique ainsi que de l'ensemble des segments. Ces mandibules ne peuvent mordre ni broyer, mais seulement collecter leur nourriture par grattage à la surface des grains de sable. Elles peuvent, à l'occasion, faire preuve sans dommage apparent d'une grande laxité. C'est ainsi qu'au cours d'un examen dans une goutte d'eau sous lamelle, le milieu s'étant presque totalement évaporé, la larve s'est trouvée littéralement écrasée, les mandibules disposées en croix de part et d'autre de la tête. Immédiatement libérée par immersion de la lame, la larve s'est montrée peu active, avec les mandibules totalement immobiles, pendant 30 minutes environ, puis elle a repris progressivement son activité normale.

Les prémandibules ( $p m a$ ), vues de face, se présentent comme une formation en $\mathrm{Y}$ sans grand modelé. Un examen dorsal les montre constituées par deux lames courtes, de direction dorso-ventrale et antéro-postérieure, qui se rejoignent au niveau du labre, en formant une tige commune brièvement prolongée vers l'arrière de la bouche (fig. 1, $\mathrm{A}^{\prime}, \mathrm{C}^{\prime}$ ). Elles sont immobiles à l'état normal. Cependant, il arrive que l'ensemble d'un tractus invisible, englobant les prémandibules et le pharynx, présente suivant l'axe de la larve quelques mouvements de translation de très faible amplitude.

Le labium (lbi), relativement court, a la forme d'une feuille dont le pétiole, situé entre les $2 \mathrm{~S}$ de l'arc génien, serait dirigé antérieurement, et le limbe postérieurement (fig. 1, C, C').

Comme pour la larve de $L$. irritans, aucun sclérite susceptible d'être assimilé à une maxille n'a été observé. La présence de la petite formation en esquille et de 3 macules noires sur le bord de la « lèvre » (voir plus loin), dans l'angle postéro-latéral de l'arc génien, c'est-à-dire à peu près dans la situation où elle a été décrite par J. W. H. Lawson (1951) chez les Culicoides, avaient fait penser à la possibilité d'une maxille en grande paitie membraneuse, mais cette hypothèse n'a pas été retenue.

6) Squelette pharyngien (fig. 1, B, C, A', D). Il comprend un hypopharynx et un épipharynx. L'hypopharynx (hypo) est en forme d'X à branches très inégales. Les branches antérieures sont courtes, étroites, parallèles entre elles, coudées à angle aigu vers la face ventrale à leur extrémité. Les branches postérieures sont au contraire très longues, fortes, légèrement coudées vers la face dorsale et appliquées sur le côté externe correspondant de l'épipharynx où elles se terminent. La formation membraneuse émanant de l'hypopharynx, décrite chez $L$. irritans, n'a pas été observée ici. L'épipharynx est constitué par un anneau ouvert do:salement, épaissi à la base, légèrement évasé à ses extrémités libres. La base porte une formation membraneuse striée, ayant l'apparence d'un très grand peigne à dents nombreuses, longues, étroites, serrées, bien visibles sous tous les angles d'observation (sauf fronto-dorsal), dirigées vers la partie postérieure de l'anneau. Sur cette face postérieure se trouvent deux petites sclérites linéaires isolés, coudés, disposés en $\mathrm{V}$ (post). L'épipharynx peut présenter trois sortes de mouvements de très faible amplitude : un mouvement passif de translation suivant l'axe de la larve vu précédemment, un mouvement en tenailles tendant à rapprocher les extrémités libres de l'anneau et un mouvement de bascule vers l'hypopharynx.

Immédiatement après la mue, l'ensemble du squelette céphalique est invisible, mais la pigmentation réapparaît très rapidement. Lorsque la larve bouge, ce squelette peut 
subir de très légers déplacements, en bloc, à l'intérieur de l'extrémité céphalique, et celle-ci peut elle-même être «télescopée » dans le mésothorax. Dans ce cas, la base des tiges ventro-latérales se trouve placée au ras du bord postérieur du mésothorax, tandis que le bord antérieur du même segment affleure l'œil ou le recouvre partiellement.

\section{II. - Formations SENSORIELles ou PRÉsumÉEs TElles.}

Elles sont nombreuses et variées; les unes sont propres au segment céphalique, les autres sont disséminées sur l'ensemble de la larve.

1) Eléments propres au segment céphalique. Ce sont les yeux, les antennes, et un groupement fronto-buccal remarquablement développé.

Les yeux (fig. 1, A, B) sont situés latéralement, au-dessous de l'extrémité coudée du bras dorso-latéral et à peu près à la hauteur de l'épipharynx ; ils disparaissent après traitement par la potasse. Les larves paraissent indifférentes à l'intensité de l'éclairage, et le passage d'une demi-obscurité à une illumination intense ne provoque aucune réaction.

Un anneau fortement sclérifié marque la base des antennes (an), en position subapicale. Largement séparées l'une de l'autre, dirigées ventralement, très courtes, elles comptent deux articles séparés par un sillon circulaire ; le premier subcylindrique, le deuxième en bouton, l'un et l'autre peu sclérifiés et légèrement aplatis dans le sens dorso-ventral. Dans sa partie postérieure et externe, ce sillon porte de minuscules papilles, au nombre de cinq ou six, disposées sur deux rangs (fig. 2, C). L'invagination de l'antenne, signalée par Smith et Lowe, n'a pu être observée.

Le groupement fronto-buccal est comparable à celui de L irritans. Les éléments qui le composent sont presque tous portés par trois formations charnues saillantes, comme turgescentes, allongées longitudinalement: l'une médiane, les deux autres latérales, qui seront appelées, par commodité, coussinets (fig. 1, B, C ; fig. 2).

Le coussinet médian $(\mathrm{cm})$ occupe la partie la plus antérieure de la tête. Il prend naissance au niveau des antennes et se termine, comme le labre qui le supporte et avec lequel il finit par se confondre par rétrécissement et amincissement progressifs, vers le milieu des mandibules. Il peut être le siège de très légers mouvements qui le font se retrousser, de la manière dont un lapin retrousse son nez. Il porte les deux séries internes, paramédianes, de sensilles numérotées de 1 à $6 \mathrm{chez} L$. irritans. Les sensilles 1 et 2 , comparables à celles observées sur le reste du tégument, se sont rapprochées, presque accolées. Elles sont situées au niveau des antennes, sur le versant dorsal du coussinet, et sont invisibles à l'examen ventral, sinon par transparence, avec mise au point profonde. La sensille 3 se présente comme un petit champignon portant quatre papilles: deux relativement grosses, disposées transversalement et deux petites disposées longitudinalement. Le plus souvent elle est invaginée, ne laissant apparaître que la partie supérieure de son chapeau. La sensille 4, impaire et médiane, porte quatre tiges grêles implantées sur un rang, contiguës, rectilignes, divergentes, transparentes, dont les plus internes sont aussi les plus longues. Les tiges externes paraissent manquer quelquefois. La sensille 5 est en forme de cône très allongé, dressé sur le tégument et 
recourbé vers la face ventrale. La sensille 6 est semblable à la précédente, mais plus longue et couchée. En raison de l'exiguïté du coussinet dans sa partie terminale, les deux sensilles 6 se trouvent rarement implantées à un même niveau ; elles sont presque toujours décalées en hauteur, et quelquefois même, très exactement situées l'une audessus de l'autre. Placées entre les deux coussinets latéraux et dirigées dans le prolongement du labre, au-dessus de l'orifice buccal, elles ne peuvent être vues en examen latéral. Les deux sensilles 5 et 6 paraissent pouvoir être respectivement assimilées aux sensilles « en club » et sétiforme de Lawson.

Chacun des éléments $\mathrm{n}^{\circ} 7$ est composé de deux très longs poils, dressés puis recourbés antérieurement, dont le plus interne et plus antérieur est généralement aussi le plus long. Insérés sur le tégument qui recouvre la mandibule, ils en suivent les mouvements et avaient déjà été signalés avec cette caractéristique par Smith et Lowe : «Immediately anterior to the mandibles are two bristles which may be easily seen when the mandibles are in motion. »

Les quatre derniers éléments sensoriels sont situés sur les coussinets latéraux $(c l)$, qui se dressent perpendiculairement au-dessus du premier sclérite de l'arc génien, et peuvent être momentanément rabattus vers l'extérieur. L'élément 8 , le plus antérieur, est semblable aux sensilles réparties sur l'ensemble de la larve ; 9 est en forme de petit champignon. Les éléments 10 et 11 sont développés en profondeur et seule leur extrémité est visible ; 10 se présente sous la forme d'une courte tige portant plusieurs papilles à son extrémité, tandis que 11 rappelle l'élément homologue de $L$. irritans mais en beaucoup plus court. Il est possible que les sensilles 10 et 11 puissent se dévaginer dans certaines conditions, mais nous ne l'avons pas observé.

D'autres formations turgescentes, de dimensions bien moindres que les précédentes, recouvrent la partie postérieure de l'arc génien. Elles sont nombreuses, disposées sur plusieurs épaisseurs, plus ou moins imbriquées, et forment une lèvre charnue proéminente $(l e v)$; quatre d'entre elles sont représentées sur la figure 2. Sur tous les exemplaires examinés, la plus externe porte trois macules arrondies, noires, disposées sur une ligne.

L'ensemble de tous ces coussinets, formations turgescentes et sensilles, est circonscrit postérieurement par un pli semi-circulaire du tégument, étroit et mince, allant d'une antenne à l'autre ( $p s c)$. Cet ensemble entoure lui-même la bouche, à demi-fermée par un diaphragme $(d p h)$ dont la partie la plus antérieure entre en contact avec l'extrémité postérieure du labre et du coussinet médian, où se trouvent les deux sensilles $\mathrm{n}^{\circ} 6$. De part et d'autre de celles-ci, le bord libre du diaphragme est prolongé par une collerette $(\mathrm{col})$ de très nombreux poils longs, gros, rectilignes, entourant l'orifice buccal proprement dit $(o b)$.

2) Formations sensorielles disséminées. Tous les segments portent des éléments se présentant sous la forme de petits disques très légèrement proéminents, centrés par une soie dressée. Les disques ont presque tous le même diamètre, $2 \mu$, mais certains sont de moitié moins larges. Les soies, subégales, mesurent de 8 à $10 \mu$ en moyenne, et jusqu'à 20-25 $\mu$ sur le segment anal. Elles sont le plus souvent simples, rarement doubles ou triples, quelquefois à branches multiples dichotomisées sur le segment 
anal. Elles disparaissent après traitement par la potasse, ce qui explique qu'elles n'aient pas été vues sur la larve de $L$. irritans. Elles apparaissent avec une netteté particulière sur la larve vivante, anesthésiée, examinée dans l'eau avec ou sans lamelle.

Pour chaque moitié du segment céphalique (fig. 1, A, B, C; fig. 2) on compte trois éléments antérieurs, à hauteur du pilier frontal et de la bouche, et sept postérieurs, disposés suivant une ligne oblique depuis la face dorsale jusqu'au pharynx. L'ensemble de la première série forme derrière la bouche un cercle complet, pouvant être

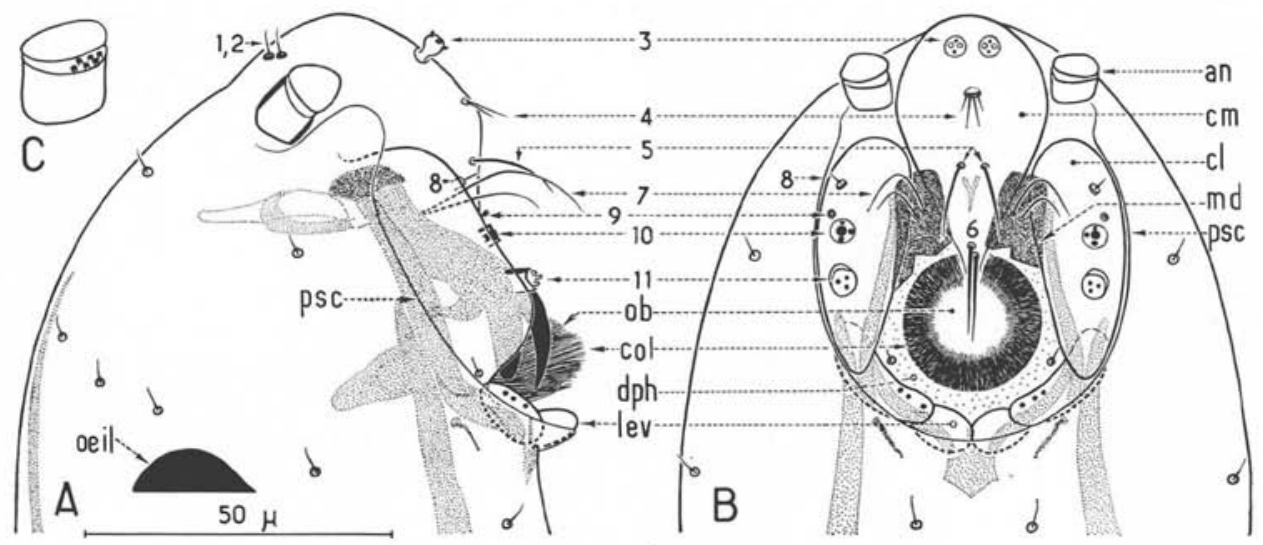

FIg. 2. - Larve de Leptoconops kerteszi K. Disposition des coussinets et des sensilles fronto-buccales; A : vue latérale; B : vue ventrale; C: vue postérieure de l'antenne droite (grossie deux fors) montrant six papilles. an: antenne. $\mathrm{cl}$ : coussinet latéral. $\mathrm{cm}$ : coussinet médian. $\mathrm{ccl}$ : collerette buccale. $d p h$ : diaphragme buccal. lev : lèvre. $m d$ : base de la mandibule (supposée sectionnée). psc: pli semicirculaire

assimilé au cercle $t$ - $w$ - $O$ de Lawson, et l'ensemble de la deuxième série un autre cercle postérieur au précédent, mieux fourni que son homologue $k-u-v$. Le prothorax ne porte que quatre éléments ventraux. Le mésothorax porte six éléments dorsaux, un élément sur chacune des faces latérales, deux éléments ventro-postérieurs, plus deux autres ventro-médians, quadruples ou cruciformes, déjà décrits chez L. irritans au même endroit. On retrouve des éléments semblables à ces derniers, et dans la même situation, sur le métathorax et le premier segment abdominal.

Tous les autres segments portent un nombre peu élevé de sensilles simples, mais leur répartition n'a pas été recherchée, sauf pour le dernier.

\section{III. - Segment anal (fig. 3).}

Il comprend deux lobes dorso-latéraux et un lobe ventro-médian, entre lesquels s'ouvre l'anus. Les deux lobes dorsaux ne sont nettement séparés qu'à leur extrémité ; 
ailleurs, ils sont unis par un pont membraneux. Un pont semblable unit également chacun des lobes dorsaux à la face latérale correspondante du lobe médian. L'anus paraît contenir deux lobes cylindriques étroits, ou papilles, faisant exceptionnellement issue à l'extérieur. Les soies sensorielles du segment anal peuvent être beaucoup plus longues que sur le reste du corps et comporter un nombre de branches plus élevé. Certaines soies, situées à la limite de deux faces, peuvent donner lieu à des interprétations différentes. Plutôt que de les décrire, il nous a paru préférable d'indiquer
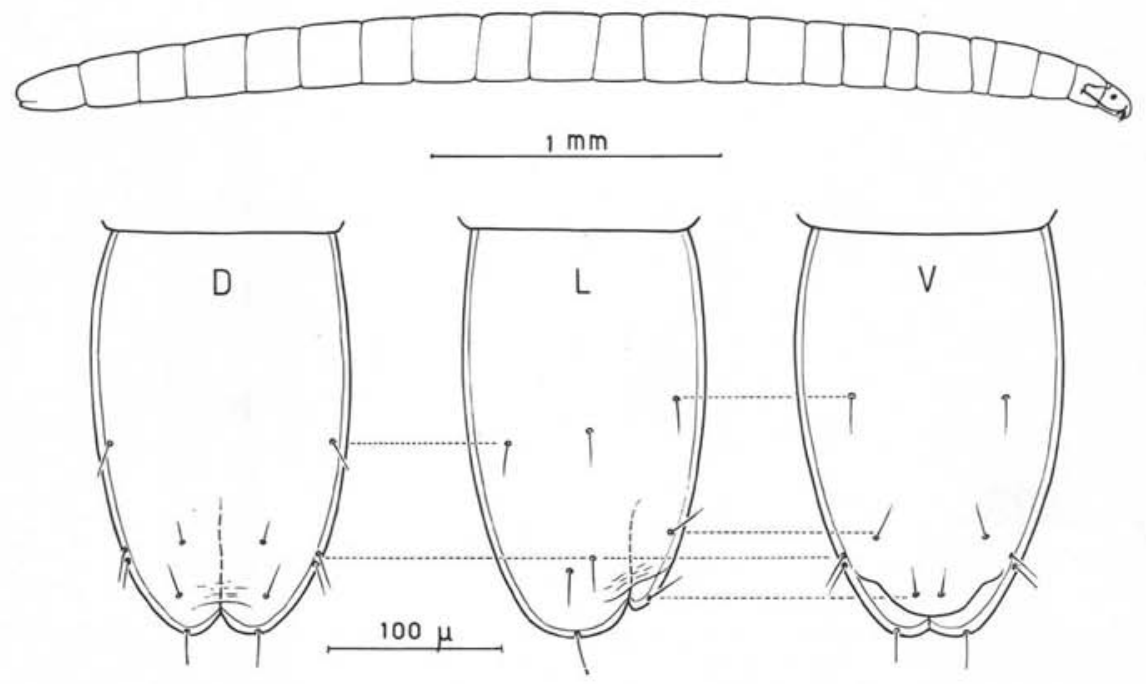

Fig. 3. - Larve de Leptoconops kerteszi K. En haut, la larve vue de profil. En bas, segment anal en vues dorsale (D), latérale (L) et ventrale (V).

leur répartition sur la figure 3, avec la correspondance de certaines d'entre elles, suivant la face examinée.

Nymphe (fig. 4).

Longueur moyenne : $2,5 \mathrm{~mm}$; elle peut être sensiblement diminuée lorsque l'abdomen se trouve en état de contraction. Comme chez les autres Nématocères et les autres Cératopogonides, elle comprend un céphalothorax et un abdomen. Les homologies avec la nymphe de ces derniers, plus particulièrement les Culicoides, sont beaucoup plus évidentes que chez la larve, tout au moins en ce qui concerne le céphalothorax. Sauf indication contraire, elles sont établies en référence aux publications de H. F. Carter, A. Ingram et J. W. S. Macfie (1920) et de Lawson (loc. cit.).

Le céphalothorax occupe, pour sa plus grande part, le cinquième antérieur de la nymphe ; il déborde ventralement sur l'abdomen jusqu'au milieu du deuxième seg- 
ment et même au-delà. Beaucoup plus ramassé que chez les Culicoides, il peut être considéré comme très grossièrement cubique, offrant à l'examen cinq faces : ventrale, frontale, dorsale et deux latérales.

La face ventrale (fig. 4, A) préfigure l'insecte parfait et à cet égard pourrait être divisée en deux parties, l'une antérieure, céphalique, l'autre postérieure, thoracique. Mais cette séparation n'est pas absolue, les gaines des pattes pro et mésothoraciques empiétant sur la région céphalique, tandis que celle de l'antenne s'insinue entre les mêmes appendices et le fourreau alaire; elle ne sera donc pas retenue, la face ventrale sera considérée dans son ensemble, de l'avant vers l'arrière. On y reconnaît d'abord les étuis antennaires (an), grossis au niveau du torus (tor), qui en dessinent les limites anté-

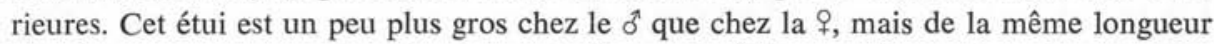
pour les deux sexes, se terminant sous le fourreau alaire, au niveau des plaques coxales mésothoraciques (voir plus loin). Entre la base des deux étuis antennaires, une formation impaire et médiane, massive et très mal délimitée sous cet angle de vue, la plaque clypéale (cly), qui recouvre le clypeus de l'adulte, ainsi que la trompe et les différentes pièces buccales déployées en éventail. Son extrémité, beaucoup mieux délimitée, recouvre la gaine des palpes sur la ligne médiane. A l'extérieur de cette plaque, la moitié ventrale de la plaque oculaire $(o c)$ munie de trois petits appendices: interne, externe, postérieur. (La plaque oculaire est divisée en deux moitiés, l'une frontale, l'autre ventrale, par l'étui antennaire). Au-dessous des deux plaques précédentes et dans la partie médiane, la gaine des palpes ( $\mathrm{pal}$ ) dessinant un $\mathrm{m}$ dont le jambage médian, raccourci, représente l'extrémité distale de la plaque clypéale. S. M. Dzhafarov (1962) note, chez la nymphe de $L$. (L.) bezzii muganicus Dzhaf., la présence de soies ventrolatérales et ventro-médianes (selon la définition des auteurs britanniques déjà cités). Nous ne les avons pas observées sur la nymphe de $L$. kerteszi, malgré une recherche attentive. Seule la protubérance postérieure de la plaque oculaire porte sur son côté interne une petite tache arrondie, claire, paraissant nue, homologue possible de la soie ventro-latérale (signalée par le chiffre 8 , en A, sur la figure), mais qui ne paraît pas constante.

Postérieurement à la gaine des palpes, les plaques coxales prothoraciques $(\operatorname{cox} 1)$ fortement convexes, et les plaques coxales mésothoraciques ( $\operatorname{cox} 2$ ) légèrement en retrait, suivies, sur leur côté externe, par la gaine de la patte correspondante ( $p 1$ et $p 2$ ). La gaine $p 1$ présente sur son bord interne, à hauteur de l'union des deux plaques précédentes, un petit diverticule suivi d'une encoche, dans lequel est logé l'éperon tibial ; p 2 passe en profondeur au-dessous de $p 1$ et l'accompagne ensuite, sur son bord externe, jusqu'à l'extrémité du fourreau alaire. La patte métathoracique prenant naissance profondément, il n'existe pas de plaques coxales correspondantes et les gaines p 3 ne sont bien visibles qu'en examen latéral. La portion terminale du fourreau alaire (aile), qui recouvre l'extrémité des trois pattes situées du même côté, forme la limite postérieure de la face ventrale.

La face frontale (fig. 4, C, G, F) représente la moitié antérieure de ce qu'il est habituellement convenu d'appeler la face dorsale ; elle nous paraît mériter une individualité propre. Passablement tourmentée, la face frontale montre dans sa partie la plus 
ventrale, au contact des étuis antennaires, un sclérite impair et médian, homologue de l'opercule des nymphes d'autres Cératopogonides, qu'en raison de ses dimensions extrêmement réduites il nous paraît préférable de nommer ici plaque frontale $(f r)$. Elle ne présente ni écailles, ni spinules, ni stries, mais porte de chaque côté une forte protubérance à l'extrémité arrondie, assombrie, munie d'une forte soie, homologue de la soie antéro-marginale des Culicoides (marquée 1 sur la figure), et un ou deux petits orifices paraissant vides. Sur son côté externe, elle prend la forme d'une lame triangulaire, marquée de deux petites taches ou papilles arrondies, claires. Cette plaque est suivie par les moitiés frontales des deux plaques oculaires $(o c)$, jointives et non séparées par l'opercule comme chez les Culicoides, où se voient trois petites éminences arrondies. Les éminences interne et postérieure portent simplement une tache arrondie claire ; l'antéro-externe une courte soie et une papille, homologues des soies antéro-dorsales des Culicoides $\left(\mathrm{n}^{\circ \mathrm{s}} 2\right.$ et 3 sur la figure), ainsi qu'une petite tache arrondie claire. Une barre transversale robuste (bar), très fortement pigmentée, faisant saillie vers l'intérieur de la nymphe, borde dorsalement les plaques oculaires dont elle épouse la courbure. A chacune de ses extrémités, marquées de trois ou quatre petites taches claires, cette barre est coudée à angle presque droit et va rejoindre un petit sclérite de la face latérale $(s d l)$ où se trouve la soie $\mathrm{n}^{\circ} 7$; ils correspondent, respectivement, au tubercule dorso-latéral des Culicoides et à la soie du même nom. La trompette respiratoire (tr) cachant ce sclérite en vue dorsale et l'extrémité coudée de la barre en vue latérale, l'un et l'autre ont été représentés en cartouche en $\mathrm{A}$ et $\mathrm{B}$ (ainsi qu'en $\mathrm{F}$ à un grossissement supérieur). Cette barre, qui marque la limite entre les régions céphalique et thoracique, est suivie dorsalement par une large bande transversale prothoracique (pro) déprimée en gouttière, qui porte latéralement les trompettes respiratoires. Il n'y a pas été vu de soie homologue de la dorso-médiane de Lawson. La limite postérieure de la face frontale précédemment définie est marquée par un pli transversal en surélévation ( $p$ tr), présentant deux petites éminences tabulaires nues (tab).

Les trompettes respiratoires (fig. 4, A, B, D) ne comptent qu'un seul article, porté sur une colonne à base très élargie. Cet article est entièrement lisse et nu, fortement pigmenté, aplati transversalement et en forme de raquette plus ou moins large et asymétrique suivant les spécimens. Il porte dans sa partie terminale deux séries contiguës de logettes très régulièrement disposées, dont l'extrémité proximale débouche dans une dilatation de la trachée, et l'extrémité distale, arrondie, fait légèrement saillie à la surface du tégument. Cette double série de «papilles» s'étend davantage sur le bord externe de l'article que sur son bord interne; à chacune de ses extrémités elle est formée d'un élément unique et médian. Longueur : 60 à $80 \mu$; plus grande largeur : $40 \mu$; épaisseur à la base : 25 à $30 \mu$.

La face dorsale (fig. 4, A, C), moitié postérieure de la face dorsale des auteurs, est essentiellement formée par deux plaques soudées sur la ligne médiane (pld), très fortement convexes dans le sens transversal, qui se prolongent, sans aucune démarcation, sur la face latérale correspondante. Chacune des plaques porte antérieurement, en position submédiane, deux petites éminences claires ; l'une interne, portant une sensille circulaire, l'autre externe, armée de deux soies fortes, longues, rectilignes, acérées 


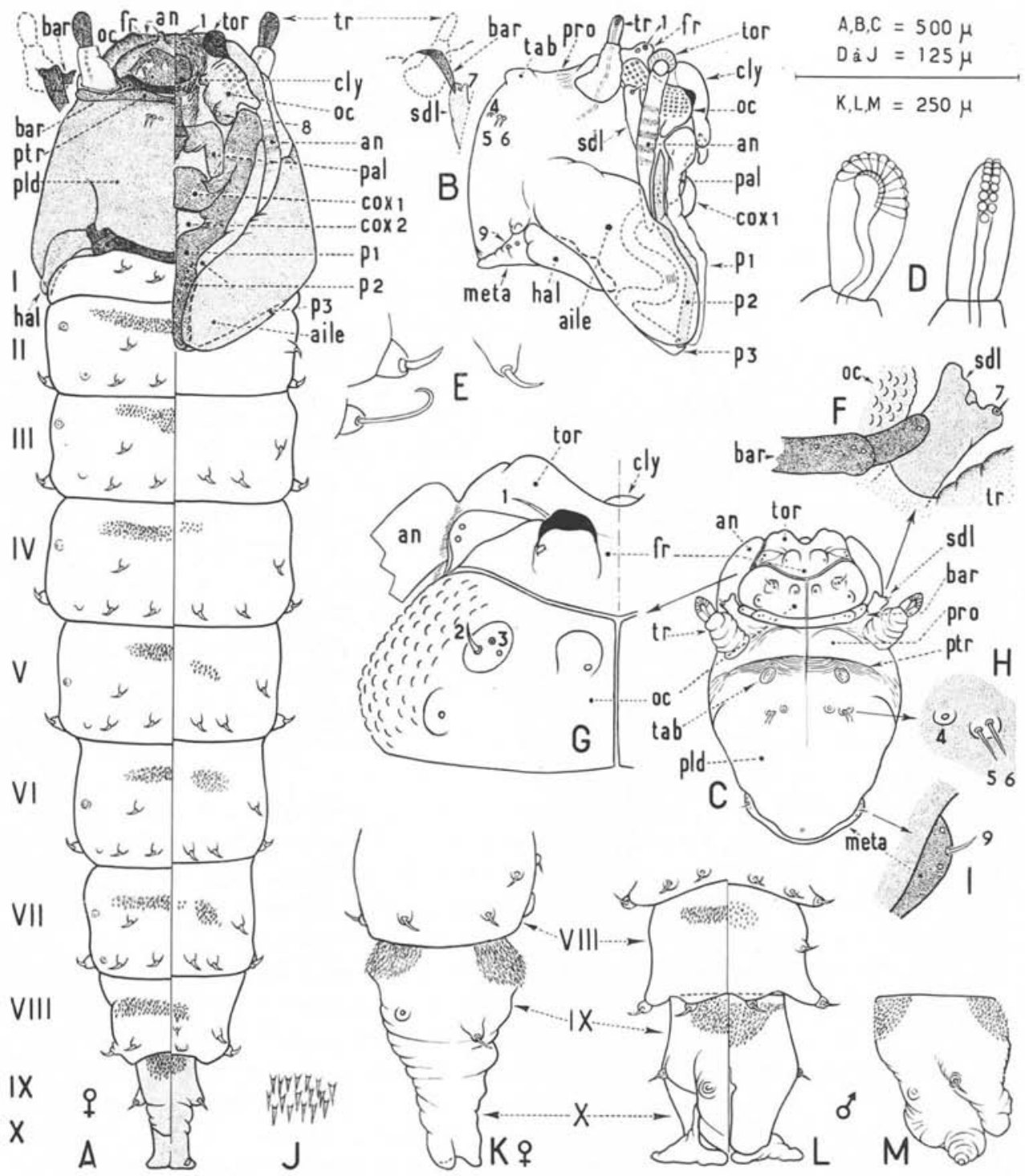

FIG. 4. - Nymphe de Leptoconops kerteszi K., ㅇ. A : nymphe en vues dorsale (à gauche) et ventrale (à droite). B: vue latérale; C: vue dorso-frontale du céphalothorax. D: trompette respiratoire droite en vues postérieure (à gauche) et latérale (à droite). E: différents aspects des crochets abdominaux. F : barre transversale du céphalothorax, soie et sclérite dorso-latéraux. G : soie des plaques frontale et oculaires. H: soies dorsales du céphalothorax. I: soie et taches claires du métathorax. $\mathbf{J}$ : épines des segments abdominaux. $\mathrm{K}$ : vue latérale des trois derniers segments abdominaux. 3 L : vues dorsale (a gauche) et ventrale (a droite) des trois derniers segments abdominaux. M : vue latérale des deux derniers segments abdominaux. aile: fourreau de l'aile. an: étui antennaire. bar: barre fronto-transversale. cly: plaque clypéale. $\operatorname{cox} 1, \operatorname{cox} 2:$ plaques coxales pro- et mésothoraciques. $f_{r}$ : plaque frontale. hal: étui de l'haltère, meta: metathorax. oc: plaque oculaire (moitié frontale ou ventrale). p1, p2, p3: gaines des pattes, pro-, méso-, métathoraciques. pal: étui du palpe. pld: plaque dorsale. pro: bande frontale prothoracique. ptr: pli fronto-transversal. sdl : sclérite dorso-latéral. $t a b$ : éminence tabulaire du pli transversal. tor : étui antennaire au niveau du torus. tr: trompette respiratoire 
(respectivement $\mathrm{n}^{\text {os }} 4,5$ et 6 ), seules représentantes du groupe des «dorsales » de Culicoides. Postérieurement prend naissance le fourreau alaire (aile).

La face latérale (fig. 4, B) prolonge sans aczident la face dorsale comme il vient d'être dit. Antérieurement, entre la base de la trompette respiratoire, la plaque oculaire et l'étui antennaire, se situe le sclérite dorso-latéral $(s d l)$, portant la soie de même nom déjà citée $\left(\mathrm{n}^{\circ} 7\right)$. Postérieurement, à travers le fourreau alaire, on aperçoit la gaine de la patte postérieure $(p 3)$, deux fois recourbée en S. L'extrémité du tibia se situe dans la deuxième courbure, entre les indications $p 1$ et $p 2$ de la figure, où quatre petits traits parallèles schématisent les dents du grand peigne. Cette gaine va rejoindre celles des deux autres pattes à l'extrémité postéro-ventrale du fourreau alaire, de telle sorte que les six griffes de l'adulte sont groupées en un même point, sur la ligne médiane de la face ventrale.

La limite postérieure du céphalothorax (fig. 4, A, B, C, I) est représentée dorsalement par le métathorax (meta), petit sclérite fortement pigmenté, étroit dans sa partie médiane, plus large à chacune de ses extrémités. Celles-ci portent une seule soie, homologue de la soie postéro-dorsale des Culicoides $\left(\mathrm{n}^{\circ} 9\right)$ et deux petites taches claires. Latéralement, les gaines des haltères ( $h a l)$ se détachent du métathorax.

Au moment de la sortie de l'adulte, la plaque frontale se sépare en bloc des plaques oculaires $(o c)$ postérieurement, des étuis antennaires (an) latéralement, et, comme l'opercule des Culicoides, se redresse en pivotant sur son bord antérieur (une amorce de séparation est représentée en G). Les deux plaques oculaires se séparent l'une de l'autre ainsi que de la barre transversale (bar) et s'écartent en pivotant sur leur bord externe. Postérieurement, jusqu'au métathorax exclu, une fente longitudinale continue sépare les deux moitiés du céphalothorax.

L'abdomen (fig. 4, A) compte dix segments de section presque circulaire, dont le diamètre décroît très régulièrement depuis le premier (500 $\mu$ environ) jusqu'au dernier $\left(80 \mu\right.$ chez la $\$, 150 \mu$ chez le $\left.\delta^{*}\right)$. Lorsque la nymphe est vivante, l'abdomen est d'une teinte légèrement plus claire que celle de la larve ; sur les dépouilles il est incolore, à l'exception des deux derniers segments qui sont très légèrement pigmentés.

Tous les segments $\left(\sigma^{\prime}, q\right)$, sauf le dernier, portent des soies habituellement courtes, fortes, rcourbées en crochet, implantées sur un tubercule saillant. Quelquefois, elles sont très longues, minces, décrivant une petite boucle à leur extrémité (fig. 4, E).

Le segment I, représenté par sa seule face dorsale, porte une rangée unique de quatre de ces crochets. De II à VIII, tous les segments portent postérieurement, sur leurs faces latérales, deux soies en crochet. De II à VII pour la face dorsale, de III à VII pour la face ventrale, chaque segment porte deux crochets antérieurs, et quatre postérieurs disposés sur un rang. Sur la face dorsale, les deux crochets les plus internes de la rangée postérieure sont plus proches l'un de l'autre que des crochets externes, et ces derniers sont eux-mêmes en position plus extérieure que le crochet antérieur correspondant; c'est l'inverse qui s'observe sur la face ventrale. Sur la face dorsale des mêmes segments se voient encore deux tubercules rudimentaires, sans crochet; l'un dans la partie moyenne, très latéral, l'autre dans l'alignement de la rangée posté. rieure. 
Le segment II est dépourvu de rangée postérieure de crochets sur sa face ventrale. VIII ne porte que deux crochets sur chacune des faces dorsale et ventrale. Chez la $\Varangle$, cette dernière porte en outre, dans sa partie médiane, deux petites soies rectilignes, ainsi que deux tubercules volumineux, mais plats et sans crochet. Sur IX ( $\left.\sigma^{\circ}, \uparrow\right)$, seules les soies ventro-latérales ont subsisté; les soies dorso-latérales sont réduites à un petit mamelon.

De II à IX dorsalement, de IV à IX ventralement, tous les segments portent à la base un amas de petites épines acérées, réparties comme il est indiqué sur la figure. D'une façon générale, elles sont plus nombreuses sur la face dorsale que sur la face ventrale.

Les deux derniers segments, soudés, renferment l'appareil génital. Très différents suivant le sexe, et beaucoup plus longs chez la $q$ que chez le $\delta$, ils sont représentés respectivement en $\mathrm{A}$ et $\mathrm{K}(\stackrel{+}{+}), \mathrm{L}$ et $\mathrm{M}\left(\sigma^{\star}\right)$.

Les nymphes dont nous avons disposé ont été obtenues par extraction du sable, en même temps que les larves. Dans ces conditions, elles se laissent couler et demeurent immergées, et immobiles, pendant des heures; même laissées au calme pendant une nuit entière, jamais nous n'en avons vu remonter à la surface. Mais aussitôt qu'elles sont déposées sur du sable humide, le quart postérieur de l'abdomen se recourbe et, par un léger mouvement circulaire, se fraie un chemin en profondeur. Plus ou moins rapidedement (de 2 à 30 minutes), la nymphe disparaît complètement, paraissant dépasser le but proposé, car peu après elle remonte et s'arrête de telle sorte que la face frontale seule affleure le niveau du sol environnant. Quelquefois, sans doute traumatisées par l'extraction, elles abandonnent après quelques essais infructueux et restent étendues sur le sable. Ce comportement initial ne permet pas de préjuger du résultat final. Des nymphes très actives sont mortes avant l'éclosion de l'adulte, tandis que d'autres considérées comme mourantes ont donné naissance à des formes ailées en parfait état. Juste avant la sortie de l'adulte, la nymphe enfouie se dresse sur le sable, où seuls demeurent les trois ou quatre derniers segments abdominaux.

\section{Technique d'isolement.}

La méthode de Smith et Lowe dite par élutriation, modifiée par J.-B. Davies et J.-R. Linley (1965) a été primitivement utilisée. L'échantillon de sable à traiter est placé dans le fond d'un cylindre en matière plastique de $35 \mathrm{~cm}$ de haut et $15 \mathrm{~cm}$ de diamètre. Grâce à un tube coudé à angle droit, maintenu à 2 ou $3 \mathrm{~cm}$ au-dessus du fond, on fait arriver tangentiellement à la paroi un courant d'eau assez violent pour créer un tourbillon ascendant de la masse liquide, susceptible d'entraîner les larves par dessus bord, sans entraîner le sable. Ce cylindre, placé sur le montage précédemment décrit pour l'isolement de la larve de L. irritans nous a donné d'excellents résultats. Cependant, l'opération est assez longue et la recherche des larves à la loupe binoculaire, parmi les nombreux débris végétaux retenus sur le tamis, reste assez absorbante. 
La technique a été modifiée de la façon suivante: 1) emploi d'un cylindre beaucoup plus grand (fût métallique à huile minérale de $62 \mathrm{~cm}$ de haut et $35 \mathrm{~cm}$ de diamètre) ; 2) tuyau d'amenée d'eau de grosse section, maintenu à $21 \mathrm{~cm}$ environ audessus du fond ; 3 ) cylindre placé sur l'étage supérieur $\left(\mathrm{n}^{\circ} 1\right)$ du montage précédemment décrit pour L. irritans; 4) eau mise en circulation «à vide », son débit étant réglé pour obtenir la turbulence nécessaire et suffisante pour entraîner les larves sans les débris végétaux (quelques essais permettent rapidement de l'apprécier) ; 5) échantillon de sable placé dans une cuvette inclinée au-dessus du cylindre; rapidement entraîné par un filet d'eau tombant dans le tourbillon près du bord, un quart de cercle au-delà de l'origine du jet, là où le flux est le plus violent en profondeur.

Cette manière d'opérer permet de jouer avec une très grande souplesse sur des différences de densité minimes. Les larves, nymphes (dans une proportion non recherchée) et Nématodes sont entraînés par dessus bord, tandis que les débris végétaux. dont la densité est légèrement supérieure, ne le sont pas; la recherche finale des larves s'en trouve grandement facilitée. D'autre part, l'espace neutre ménagé dans le cylindre au-dessous du jet, permet de traiter sans interruption une quantité importante de sable ; cet espace peut d'ailleurs être agrandi à volonté. Enfin, l'emploi d'un tamis "immergé », qui s'est montré très efficace dans la protection des larves et des nymphes contre les traumatismes de l'opération, permet d'envisager des isolements massifs en vue de recherches biologiques diverses.

\section{Bibliographie}

Carter (H. F.), Ingzam (A.) et Macfie (J. W. S.), 1920. - Observations on the Ceratopogonine Midges of the Gold Coast with descriptions of new species. Part. II. Ann. trop. Med. Parasit., 14, 211-274 et 2 pl.

Clastrier (J.), 1971. - Isolement et description de la larve de Leptoconops (Leptoconops) irritans Noé, 1905 (Diptera, Ceratopogonidae). Ann. Paras. hum. et comp., 46. 737-748.

Davies (J. B.), et Linley (J. R.), 1965. - Observations on the Breeding Sites of the Sandfly Leptoconops bequaerti in the Montego Bay area of Jamaica, with a note on one breeding site in Honduras. Caribbean Jl Sci., 5, 117-128.

Dzhafarov (Sh. M.), 1962. - Morphology of preimaginal phases of Leptoconops bezzii muganicus Dzhaf., of blood sucking sand flies (Diptera, Heleidae). Zool. Zl, 41, 241-246.

LAwson (J. W. H.), 1951. - The anatomy and morphology of the early stages of Culicoides nubeculosus Meigen (Diptera, Ceratopogonidae $=$ Heleidae). Trans. $R$. entom. Soc. Lond., 102, 511-574, 1 pl.

Rioux (J.-A.) et Descoux (S.), 1965. - Détection du biotope larvaire de Leptoconops (Holo. conops) kerteszi Kieffer, 1908 (Diptera, Ceratopogonidae), dans le Midi méditerranéen. Ann. Paras. hum. et comp., 40, 219-229.

Sмith (L. M.) et Lowe (H.), 1948. - The Black Gnats of California. Hilgardia, 18, 157. 183. 